\title{
Fast electrical characterization with low hardware requirement
}

\author{
Uwe Pliquett ${ }^{1,2,3}$ \\ 1. Institut für Bioprozess- und Analysenmesstechnik, Heilbad Heiligenstadt, Germany \\ 2. Section Editor, Journal of Electrical Bioimpedance \\ 3. E-mail any correspondence to: Uwe.Pliquett@iba-heiligenstadt.de
}

Electrical impedance spectroscopy can be performed using a great variety of methods and instrumentation. The most popular method, sweeping though a frequency range and measuring the impedance, either magnitude and phase or real and imaginary part at each desired frequency, offers high precision due to selective amplifiers and high dynamic range with respect to impedance magnitude. Highly sophisticated laboratory equipment guarantees robust measurement while software packages assist the interpretation of the measurement results. Although automated impedance analyzers are reasonably fast, the physical limitation of signal engagement at each measurement frequency always applies. Traditionally, the majority of the impedance analyzers are bulky and expensive and thus not suited for process instrumentation. During the last two decades, more and more economic instruments with small dimensions and low energy constraints conquer the market. Although the majority of these devices work in the frequency domain, time domain based approaches are increasingly offered.

\section{Electrical characterization in time domain}

While in the frequency domain the physical property like impedance, admittance or permittivity is a function of frequency (spectrum), its analogue in time domain is a function of time i.e. the impulse response or weight function. Practically, the time functions of current, voltage or charge as response to a broad bandwidth signal are monitored. All excitation functions other than sinusoidal signals contain more than one frequency. Especially broad-bandwidth signals such as square wave, multisine, maximum length sequences or chirps contain high energy density in a given frequency range. Tailoring signals like multisine or chirp can concentrate the total signal energy within the desired frequency range for the measurement application.

The general approach for time domain based measurements is the application of a broad bandwidth signal and monitoring the system answer. Theoretically, it is sufficient to control for instance the current and monitor the voltage or vice versa. However, due to the transfer behavior of the instrumental part of the measurement chain, it is advisable to monitor both, the voltage and the current. The separation of the discrete frequencies contained in the signals using Fourier transformation yields the amplitude and the phase spectrum of voltage and current. The quotient of Fourier transformed voltage $\mathscr{F}(u(t))$ and current $\mathscr{F}(i(t))$ yields the impedance spectrum $Z(j \omega)=\mathscr{F}(u(t)) / \mathscr{F}(i(t))$. All further processing can be done in frequency domain. It should be noted that for single measurements the excitation signal should be applied at least for 10 periods of the lowest frequency component in order to reach a steady state. For single transients without signal engagement, Laplace transformation should be used instead of Fourier transformation.

\section{Measurement speed}

An important argument for using electrical characterization in time domain is the speed of the measurement, which can be by orders of magnitude faster than sweeping through a frequency range. Although a tradeoff between speed and measurement precision is unavoidable, time domain based measurements are quit comparable to those in the frequency domain. If the speed is not the important requirement, averaging can dramatically enhance the signalto-noise ratio. Theoretically, the measurement precision will 
reach that of selective amplifiers, like for instance lock-inamplifiers, when averaging over times else required for spectral analysis in the frequency domain. Today, fast ADCs are able to oversample, thereby averaging even through single shot measurements, which makes averaging over several periods of the excitation signal unnecessary. Another important feature of measurements in time domain is the comparatively low hardware requirement. Although special integrated circuits for impedance measurements exist, virtually all microcontrollers with fast enough analog features (ADC, DAC, OPA) are suited for electrical characterization in the time domain. For single shoot measurements, the ADC-speed (samples per second) should be twice as high as the highest frequency compound in the excitation signal. A highly versatile approach, achieving a functional extent comparable to commercial network analyzers uses oscilloscopes, e.g. USB-oscilloscopes, and frontends for adaption to the electrode interface. Such solutions are increasingly introduced in scientific environments.

\section{Relaxation spectroscopy}

The impedance, i.e. the complex resistance to the movement of electric charges in a material, is a physical quantity in the frequency domain. Its analogy in time domain is the impulse response (also weight function), which is a function of time as reaction to the application of Dirac function. As by definition, a system is excited at $\mathrm{t}=0$ and relaxes either back to the state before or approaches a new state for instance when excited by a step function.

The relaxation function exhibits exactly the same information as the impedance spectrum and modeling yields the same elements. Because of the complicate generation of the Dirac function with infinite amplitude and zero time duration but having an integral of 1 (area), is the step function for relaxation studies much more popular. Since the amplitude of a step is 1 from $0<t<\infty$, the relaxation process for this kind of stimulus may not approach a constant value (e.g. capacitor charging with current step). Moreover, undamped resonance phenomena cause overshoot and slow settling. However, in case of biological objects, showing the well-known dispersions $(\alpha, \beta, \gamma)$ overshoot does not occur, at least not caused by the MUT. Each Debye-relaxation $y_{i}(t)$ causes an exponential decay in time domain with relaxation strength $A_{i}$ and a time constant $\tau_{i}: \quad y_{i}(t)=A_{i} e^{-t / \tau_{i}}$. The sum of all individual relaxations is the time function measured. As in the frequency domain, involvement of electrodes or the appearance of non-ideal elements yields more complicate functions. The Cole model or a CPE describing electrode behavior yield a distribution of relaxation times or an exponential with fractional coefficient, which is the analogue to depressed semicircles in the impedance spectrum.
Hardware requirement

Impedance measurement employs sinusoidal excitation while the magnitude and phase or real and imaginary part are measured. High precision and effective noise suppression is achieved by using phase sensitive rectifiers, lock-in-amplifiers or self-balancing bridges. Alternatively, both current and voltage can be traced and Fourier transformed. Using the first Fourier-coefficient (fundamental frequency) enables impedance calculation at the applied frequency.

In the time domain, a simple switch or digital output can be used for generating the stimulus while the data acquisition system traces voltage and current.

Instrumentation designed for measurements in both domains - frequency and time domain - utilize front ends for adapting the instrument to 2-, 3-, or 4-electrode systems or use multiplexers for multi-electrode systems. Basically, these front ends are the same for both approaches.

Single shot broadband measurements are compatible with modern techniques. Even the acquisition of 6 or more frequency or time decades, which require at least 2.000.000 time instants, does not cause problems. However, if resources are limited or continuous monitoring is required, relaxation spectroscopy can pose considerable advantages.

Given the fact that the response time function after step excitation for typical bioimpedance applications are exponential or square root of time function (Warburg impedance), allows simple data reduction by fast sampling after the step occurred while dramatically slowing down when time elapsed. To avoid aliasing but also for noise reduction, partial integration with successively increasing intervals is used. Thus, only 3 to 10 time instants per time decade are necessary without comprising the measurement accuracy as shown for several practical applications.

With the absence of huge data streams, hardware can be reduced to a microcontroller with integrated analog-digitalconverter and if necessary - an analog frontend. Moreover, the marginal data volume allows a low bandwidth for data transmission, which paves the way for continuous monitoring. The comparatively high effort for data processing poses no problem since it is done in an adequate powerful machine.

\section{Applications}

The use of relaxation spectroscopy can access new applications. Especially the measurement speed, the ability for continuous monitoring, simple hardware and low energy constraints makes it interesting for applications where cheap instrumentation is favored.

It is possible to extent RFID-systems with a complete impedance spectrometer which is powered by the RFID-chip and reads out the spectrum, for instance from $100 \mathrm{~Hz}$ to 1 $\mathrm{MHz}$ within $100 \mathrm{~ms}$. Very simple systems in the extreme case with only one resistor as external compound are proposed 
for single use applications. Fast systems requiring fast hardware as well have been tested for impedance cytometer while the principle of relaxation spectroscopy has been tested for complete impedance spectroscopy at 10.000 individual electrodes with a readout-time of all spectra from $1 \mathrm{kHz}$ to $100 \mathrm{kHz}$ within less than a second. Electrochemical applications for biosensors based on binding of substances, for instance to aptamers are robust and have been tested to deliver the same spectra as commercial equipment used as reference. For embedded applications where the impedance spectrum is not needed, decisions can be made on the basis of relaxation times and relaxations strengths but also distribution factors directly obtained from relaxation measurement without transformation into the frequency domain.

\section{Conclusion}

Relaxation spectroscopy is an old technique, which poses the advantage of low prized hardware with low energy consumption for applications where laboratory equipment is too expensive or requires too much energy for continuous operation. The need for obtaining complete spectra reaches theoretical limits while the measurement precision is sufficient for most applications.

Making robust measurements based on relaxation spectroscopy needs good understanding of the basics in order to avoid or minimize error sources like high frequencynoise, $50 \mathrm{~Hz}$-crosstalk, drift of base lines or jitter in time instants. 